\title{
Questes
}

\section{Le temps de la fête}

\section{La fête imprévue : entrées royales et solennelles à Lyon (1460-1530)}

\section{Tania Lévy}

\section{(2) OpenEdition}

\section{Journals}

\section{Édition électronique}

URL : http://journals.openedition.org/questes/4269

DOI : 10.4000/questes.4269

ISSN : 2109-9472

\section{Éditeur}

Les Amis de Questes

\section{Édition imprimée}

Date de publication : 31 octobre 2015

Pagination : $33-44$

ISSN : 2102-7188

\section{Référence électronique}

Tania Lévy, «La fête imprévue : entrées royales et solennelles à Lyon (1460-1530) », Questes [En ligne], 31 | 2015, mis en ligne le 24 janvier 2016, consulté le 01 mai 2019. URL : http:// journals.openedition.org/questes/4269; DOI : 10.4000/questes.4269 


\title{
La fête imprévue : entrées royales et solennelles à Lyon (1460-1530)
}

\author{
Tania LEVY \\ Université Paris-Sorbonne
}

Au tournant du XVI siècle, la ville de Lyon a accueilli à de nombreuses reprises les souverains français et étrangers ainsi que des personnages ecclésiastiques d'importance. Évidemment, les entrées royales et solennelles ne sont pas les seules occasions de travail pour peintres, sculpteurs ou orfèvres : quelques rares mystères, des tournois, les naissances royales incitent les consuls à faire appel à eux, le plus souvent pour réaliser des écussons aux armes de la ville ou de la personne fêtée. Ces occasions sont cependant moins contraignantes et moins prenantes, ainsi que parfois bien moins documentées, que ne le sont les entrées.

À Lyon, le Consulat est l'entité administrative en charge de la cité depuis le XIV ${ }^{\mathrm{e}}$ siècle. Il est formé de douze membres, parmi lesquels on retrouve souvent les mêmes noms. Parmi ses attributions se trouvent la levée des d'impôts ou encore le financement des dépenses nécessaires au fonctionnement de la ville ${ }^{1}$.

\footnotetext{
${ }^{1}$ Jean-Baptiste Monfalcon, Histoire monumentale de la ville de Lyon, Paris/Lyon, Didot/Bibliothèque de la ville, 1866, 8 vol., vol. I, cit. p. 333 ; Karine Gentil-Perret, «Le patriciat lyonnais au $\mathrm{XV}^{\mathrm{e}}$ siècle », mémoire de maîtrise sous la direction de M. Riche, Lyon III, 1993 ; Caroline Fargeix, «Les élites lyonnaises au miroir de leur langage », thèse de doctorat sous la direction de Nicole Bériou, Lyon II, 2005, p. 14 15. De nombreux documents évoquent les réparations du pont du Rhône et les fortifications de la ville : les séries BB et CC des Archives municipales de Lyon.
} 
Lyon connaît aux alentours de 1500 une situation plutôt confortable. Forte de quatre foires confirmées par Louis XI dès $1463^{2}$ et malgré l'éclipse des années $1480^{3}$, elle accueille de nombreux marchands étrangers (principalement du nord de l'Europe mais également des Italiens) ainsi que des banquiers et financiers ${ }^{4}$. Elle est alors la première place bancaire du royaume et attire dès 1466 le comptoir des Médicis, au détriment de Genève.

L'absence d'université et de parlement n'a pas empêché l'installation dans la ville en 1473 d'un imprimeur flamand, Guillaume Le Roy. Dès la fin du siècle, Lyon est ainsi le troisième centre d'édition au niveau européen, derrière Venise et Paris ${ }^{5}$. En outre, la cité tient quasiment le rôle d'une capitale dans les années 1490-1500 : les rois de France s'y installent avant de partir vers l'Italie. Les souverains et la cour séjournent donc des semaines voire des mois sur les bords de Saône pour préparer leur départ ou au retour des expéditions.

\footnotetext{
${ }^{2}$ Histoire de Lyon des origines à nos jours, dir. André Pelletier et Jacques Rossiaud, Le Coteau, Horvath, 1990, 2 vol., vol. 1, cit. p. 75. Dans l'ordonnance royale de 1463, il est d'ailleurs bien précisé que les étrangers peuvent s'établir à Lyon sans risquer de voir leur héritage perdu, même en cas d'absence de testament.

${ }^{3}$ En 1484, aux États Généraux de Tours, les foires de Lyon sont supprimées et remplacées par celles de Troyes et Bourges. Il fallut attendre 1494 pour que Charles VIII rétablisse définitivement les foires de la cité.

${ }^{4}$ Sur les marchands et les foires, voir Richard Gascon, Grand Commerce et vie urbaine au XVI siècle : Lyon et ses marchands, Paris, SEVPEN, coll. « Civilisations et sociétés », $\mathrm{n}^{\mathrm{0}}$ 22, 1971, 2 vol. ; Jacqueline Boucher, Présence italienne à Lyon à la Renaissance. Du milieu du XV à la fin du XVI siècle, Lyon, Lugd, 1994 ; Giuseppe Iacono, Salvatore Ennio Furone, Les Marchands banquiers florentins et l'architecture à Lyon au XVI siècle, Paris, Publisud, coll. «La France au fil des siècles », 1999.

${ }^{5}$ André Pelletier et Jacques Rossiaud, op. cit., cit. p. 23. Le premier ouvrage sorti des presses lyonnaises fut le Compendium breve du cardinal Lothaire devenu pape sous le nom d'Innocent III, le 17 septembre 1473. Voir Henri-Jean Martin, «Le rôle de l'imprimerie lyonnaise dans le premier humanisme français », dans L'humanisme français au début de la Renaissance, actes du colloque international de Tours, Paris, Vrin, coll. «De Pétrarque à Descartes », vol. 29, 1973, p. 81-91 et Lyon l'humaniste. Depuis toujours ville de foi et de révoltes, dir. Claude Royon, Paris, Autrement, coll. « Mémoires », vol. 105, 2004, p. 68.
} 


\section{La fête imprévue}

Le phénomène de l'entrée royale, connu dès le début du XIV siècle, a été largement étudié par les historiens depuis la seconde moitié du $\mathrm{XX}^{\mathrm{e}}$ siècle $^{6}$. Il s'agit d'une cérémonie à la gloire du roi mais également à la gloire de la ville, souvent mise en scène et exaltée dans les décors et les histoires. L'élément religieux y tient une place prépondérante, ce que la présence du dais (sous lequel prend place l'hôte de marque) affirme avec force ${ }^{7}$. Les entrées lyonnaises les plus anciennes présentent d'ailleurs uniquement des scènes hagiographiques ou bibliques, ainsi pour celle de Louis XI en 1476. Lors de sa déambulation dans les rues de la ville, la personne accueillie admire

\footnotetext{
${ }^{6}$ Bernard Guenée et Françoise Lehoux, Les Entrées royales françaises de 1328 à 1515, Paris, Éditions du CNRS, coll. « Sources de l'histoire médiévale », n 5, 1968 ; Joël Blanchard, «Le spectacle du rite: les entrées royales », Revue Historique, $\mathrm{n}^{\mathrm{o}}$ 627, t. CCCV/03, 2003, p. 475-519; Élodie Lecuppre-Desjardin, La Ville des cérémonies. Essai sur la communication politique dans les anciens Pays-Bas bourguignons, Turnhout, Brepols, coll. «Studies in European urban history », vol. 4, 2004. Parmi la bibliographie abondante consacrée à ce thème, voir également : Lawrence M. Bryant, The King and the city in the parisian royal entry ceremony, Genève, Droz, coll. «Travaux d'humanisme et Renaissance », $\mathrm{n}^{\circ} 216,1986$ et du même auteur, Ritual, ceremony and the changing monarchy in France, 1350-1789, Farnham, Ashgate, coll. «Collected studies series», nº37, 2010; Philippe Lardellier, Les Miroirs du paon. Rites et rhétoriques politiques dans la France de l'Ancien Régime, Paris, Champion, coll. «Études et essais sur la Renaissance », $\mathrm{n}^{\mathrm{o}}$ 44, 2003 ; Fabienne Joubert, «Les tableaux vivants et l'Église » dans Le Théâtre de l'Église (XII $-X V I^{e}$ siècles), dir. Marie Bouhaïk-Gironès, Paris, LAMOP, 2011, en ligne sur https://lamop.univ-paris1.fr/IMG/pdf/FJoubert.pdf [10.11.2014].

7 Élie Konigson, L'Espace théâtral médiéval, Paris, Éditions du CNRS, coll. «Le Chœur des muses », 1975, p. 198 ; Robert Walter Scheller, «Imperial themes in art and literature of the early French Renaissance: the period of Charles VIII ", Simiolus : Netherlands Quarterly for the History of Art, vol. 12, $\mathrm{n}^{\mathrm{o}} 1$ 1, 1981-1982, p. 5-69, cit. p. 15 et «Ensigns of authority: French royal symbolism in the age of Louis XII », Simiolus : Netherlands Quarterly for the History of Art, vol. 13, $\mathrm{n}^{\mathrm{o}} 2$, 1983, p. 75-141, cit. p. 102 ; Franck Collard, «Fête du prince ou Fête de Reims : l'entrée de Charles VIII à Reims le 29 mai 1484 »dans Fêtes et politique en Champagne à travers les siècles, dir. Sylvette Guilbert, Nancy, Presses Universitaires de Nancy, coll. «Études champenoises», 1992, p. 59-71, cit. p. 62 ; Cora Dietl, «A Corpus Christi play as part of the Habsburg monarchy's politics », European Medieval Drama, vol. 6/6, 2003, p. 109-119.
} 
décors et histoires (scènes mouvantes, tableaux vivants) situés à certains endroits précis, qui ne varient guère.

La question qui se pose en premier lieu est le bouleversement induit par l'entrée dans le temps urbain. Comment les consuls et les artistes y font-ils face ? Cela affecte-t-il leur quotidien et leur façon de travailler?

Les entrées lyonnaises présentent un schéma d'organisation constant ${ }^{8}$. Une fois informés de l'arrivée imminente de leur hôte, les consuls nomment généralement plusieurs personnes pour s'occuper de l'événement, parmi les notables ou dans leurs propres rangs. Ils lèvent ensuite un impôt exceptionnel spécialement destiné à couvrir les frais de l'entrée. Cette organisation n'est pas toujours la même selon les villes : à Avignon, ce sont les taxes habituelles de la cité qui servent à la préparation de ces fêtes ${ }^{9}$.

Le temps de préparation d'une entrée varie grandement en fonction des cas : en 1476, les histoires sont choisies dès le 13 février, soit plus d'un mois avant l'arrivée de Louis $\mathrm{XI}^{10}$. En 1485, les consuls apprennent le 6 décembre la prochaine venue du cardinal-archevêque, Charles II de Bourbon, qui fait son entrée le 22 du même mois ${ }^{11}$. En

\footnotetext{
${ }^{8}$ Vingt entrées ont donné lieu à la préparation de mystères ou à l'embauche de peintres : en 1463, 1468, 1476, 1480, 1485, 1489, 1490, 1494, 1495, 1498, 1499, 1500, 1503, 1507, 1509, 1515 (deux entrées), 1516, 1518 et 1528. La venue de la reine Éléonore et du dauphin en 1533 n'entre pas dans les bornes chronologiques de cette recherche mais sera convoquée pour comparaison. Cinq autres entrées sont mentionnées mais ne sont pas accompagnées de décors : 1463 (duc de Savoie), 1466 (Charles II de Bourbon), 1494 (le légat pontifical André d'Espinay), 1502 (la future reine de Hongrie) et 1518 (le duc d'Urbino). De nombreux personnages sont également accueillis à Lyon par des présents et la révérence des consuls, sans qu'une entrée ne soit projetée : voir A. M. Lyon, série BB.

${ }^{9}$ Mathieu Bonicel, «Les modes de financement public des performances à Avignon à la fin du Moyen Âge », Studies in Early Modern France, vol. 13, «Spectacle », 2010 , p. 3140.

${ }_{11}^{10}$ A. M. Lyon, BB13, $\mathrm{f}^{\mathrm{o}} 37$ : le roi fait son entrée le 22 mars.

${ }^{11}$ A. M. Lyon, BB15, $\mathrm{f}^{\mathrm{o}} 339-342$.
} 
1490, le roi leur écrit à peine une semaine à l'avance pour les prévenir de sa prochaine arrivée. En revanche, les consuls ont beaucoup de temps pour préparer à l'avance l'entrée de la reine Claude en 1516 : dès le 30 octobre 1515, ils chargent Jean Richier et Jean Yvonnet de penser à des mystères $^{12}$. La reine n'accepte cependant aucun honneur tant que le roi n'est pas de retour d'Italie ${ }^{13}$ et ne fait son entrée en ville que le 2 mars $1516^{14}$. Le manque de temps influe rarement sur le choix des histoires et le déroulé de l'entrée ; ce n'est que le manque de deniers qui préoccupe les consuls, même quand ils n'ont qu'une semaine de délai.

En 1502, pour la future reine de Hongrie ${ }^{15}$, les consuls se contentent de parer la ville et de lui faire un don en argent « a cause de la bresveté du temps et que [...] ne seroit possible y faire chose qui fust plaisant a icelle damme et dont la ville peust avoir honneur ${ }^{16} »$. Dans ce cas précis, il est fort possible que, outre la brièveté du délai, la qualité de la personne accueillie ait eu une importance dans le choix de faire ou non une entrée. Il arrive également que le roi annule son entrée ou bien la retarde et que la mise en place des décors soit alors bouleversée, comme en $1499^{17}$. À cette date, deux ouvriers durent dégarnir une porte et la regarnir le jour de l'entrée ; on ne connaît pas les détails ni les raisons de ce retard qui ne semble pas perturber la préparation de la fête. Ce délai n'occupe en effet qu'une seule ligne des comptes.

${ }^{12}$ A. M. Lyon, BB34, $\mathrm{f}^{\mathrm{o}} 100 \mathrm{v}$.

${ }^{13}$ A. M. Lyon, BB34, $\mathrm{f}^{\mathrm{o}} 106$.

${ }^{14}$ A. M. Lyon, BB34, $\mathrm{f}^{\mathrm{o}} 147 \mathrm{v}-164 \mathrm{v}$ et CC666, $\mathrm{f}^{\mathrm{o}} 27-43 \mathrm{v}$.

${ }^{15}$ Anne de Foix, sur la route la conduisant en Hongrie afin de célébrer son union avec le roi Vladislas IV de Bohême.

${ }^{16}$ A. M. Lyon, BB356, $\mathrm{f}^{\mathrm{o}} 23$. L'annonce de sa venue arrive le 3 juin, alors que la lettre du roi est datée du 20 mai ; la reine fait son entrée le 7 juin. Or le duc de Savoie prévient de sa venue le 26 mars, pour une entrée le 30 mars et les consuls font le nécessaire pour faire une entrée.

${ }^{17}$ A. M. Lyon, CC538, pièce $\mathrm{n}^{\mathrm{o}} 2, \mathrm{f}^{\mathrm{o}} 13$ : «Aux gaignedeniers qui garnirent lad. porte de Bourneuf et desgarnirent pour ce que le roy ne vint si tost et regarnirent le jour de l'entree ». 
Outre les responsables financiers du projet, un ou plusieurs personnages, parfois distincts des organisateurs, doivent proposer des idées. Il n'est laissé généralement que très peu de temps à ces derniers pour y penser : une nuit ou une journée doivent suffire à présenter un canevas de la fête. Cependant, dans le discours des artistes ou des concepteurs, cette rapidité demandée ne semble pas poser de problème. On pourrait multiplier les exemples mais l'astrologue Simon de Phares, sollicité pour deux entrées en 1485 et 1490 , l'exprime bien : «j'ay ceste nuyt passee fait le gect au plus pres que j'ay peu et pensé en deux choses dont hier me donnastes charge principallement ${ }^{18}{ }$.

Le discours des artistes, quand il est conservé, est en ce sens parlant. Prenons celui du Lyonnais Jean Perréal, peintre des rois Charles VIII, Louis XII et François I ${ }^{\mathrm{er}}$. En 1494, il écrit au Consulat une longue lettre pour se plaindre de ne pas avoir été payé de la dernière entrée qu'il a organisée la même année. Entre deux réclamations, Perréal livre de précieuses informations sur les préparatifs de l'événement: il prend sa mission « cœur au ventre » et se charge de courir partout,

de conduire, ordonner, mettre gens en oeuvre que d'aler sur le Rosne puis en Bourgneuf plus au Cordeliers puis a l'ostel de la ville et tous les jours prés de 25 personnes a respondre de heure en heure faittes cecy, faittes cela puis paier puis escripre puis penser et regarder se tout yront bien et de tout luy tout seul tenir compte ${ }^{19}$.

Il a travaillé en tout soixante-six jours, dit-il, dont huit après l'entrée pour dresser les comptes. Il s'agit donc d'un long travail, lourd à assumer, car Perréal est en charge de toute l'entrée, de l'invention du canevas à la supervision des artistes. Pourtant, le peintre n'évoque pas de

\footnotetext{
${ }^{18}$ A. M. Lyon, CC556, pièce $\mathrm{n}^{\mathrm{o}} 3$.

${ }^{19}$ A. M. Lyon, CC527, pièce $n^{0} 4$.
} 
difficulté particulière due aux délais ; mais il se plaint plutôt de la charge de travail - et du fait de n'avoir pas été payé.

Il faut souligner un point caractéristique de la ville de Lyon : les réalisations créées par les peintres à ces occasions sont prises en compte dans le texte des Statuts de la communauté des peintres, sculpteurs et peintres verriers, octroyés par Charles VIII en 1496. Ce texte fondamental compte cinquante-deux articles qui règlementent la pratique des métiers, de façon très approfondie (matériaux, accès à la maîtrise, etc.). L'article 47 précise ainsi que si les peintres, sculpteurs et verriers ont « chose hastive a faire de leurs ars, comme a entrees de roys, roynes, princes ou seigneurs $[\ldots]$ » et n'ont pas l'occasion de respecter à la lettre le règlement, ils «ne seront pour lors reprins de ladicte besongne ne emendables $^{20} »$. Cette exception consentie dans le cadre des entrées est exceptionnelle : on ne la rencontre dans aucun autre texte d'ordonnance en France à la fin $d u X V^{\mathrm{e}}$ siècle. Elle souligne la place majeure que les fêtes occupent dans la vie artistique de la cité ${ }^{21}$ mais également la rapidité nécessaire à la préparation des entrées, qui s'accorde mal avec le temps réglementaire du travail ordinaire.

\section{La temporalité de l'entrée}

Le plus souvent, l'hôte arrive par la porte de Bourgneuf, située au nord de la ville sur la rive droite de la Saône. Il traverse ensuite le quartier dit de Fourvière et arrive par la grand-rue à la cathédrale, où le clergé prend le relais des consuls et des habitants. Après la cérémonie religieuse, l'arrivant rejoint son logement (dont le lieu varie

\footnotetext{
${ }^{20}$ Marius Audin et Eugène Viala, Dictionnaire des artistes et ouvriers d'art du Lyonnais, Les éditions provinciales, $1992^{2}\left(1918-1919^{1}\right)$, p. XXVI.

${ }^{21}$ Jean Jacquot, Les Fêtes de la Renaissance, vol. 2 «Fêtes et cérémonies au temps de Charles Quint », Paris, Éditions du CNRS, coll. «Le chœur des muses », 1973, cit. p. 10.
} 
continuellement). Le soir-même ou le lendemain, un banquet est donné dans ce logis et les consuls offrent alors à l'hôte le cadeau qu'ils ont préparé pour lui.

Mais comment se déroule exactement le temps de l'entrée ? Accueilli à l'extérieur des murailles par la délégation des consuls, l'hôte prend place sous un dais porté par quatre membres du Consulat. Souvent, une première histoire est organisée avant même la première porte. Le cortège se forme ensuite pour déambuler dans les rues, tendues de tapisseries et jonchées de fleurs et de feuillages. La chevauchée dans les rues de la ville est ponctuée de plusieurs saynètes, appelées échafauds, devant lesquelles le roi ou le prince s'arrête. C'est là que la durée de l'entrée prend tout son sens et surtout pose problème. On ne possède en effet aucune donnée sur le temps passé dans les rues et devant chaque histoire. Pourtant, il est certain que le cortège faisait de fréquents arrêts devant ces échafauds et que la personne accueillie était parfois mise en scène et prise à partie par les acteurs. C'est son déplacement qui déclenche la parole des personnages ou la mise en marche des effets spéciaux, car le scénario des entrées se concentre autour d'elle. Le personnage fêté est donc spectateur et acteur tout à la fois ${ }^{22}$ : il assiste aux histoires organisées en son honneur mais il en est souvent également le protagoniste. Ainsi la temporalité de l'entrée est aussi et peut-être d'abord celle du déplacement de l'hôte. En 1490 par exemple, l'avancée de Charles VIII vers le premier char, celui consacré aux signes du zodiaque, est le moteur de la révélation du mystère. La roue des douze signes, en mouvement jusqu'alors, s'arrête pour que le soleil qui en forme l'axe désigne le signe du lion. Hormis le roi, ses proches groupés autour de lui et une partie du cortège, le mouvement de la scène reste

\footnotetext{
22 Joël Blanchard, op. cit., 2003, p. 477-478.
} 
sans doute invisible au reste de la suite royale. En effet, dans ce cas précis mais également pour les autres histoires, la vision de l'échafaud sans mouvement, sans parole, ne permet pas, a priori, d'en comprendre le sens et l'intérêt. Est-il possible alors que les saynètes aient été jouées plusieurs fois?

Il faut pour le comprendre évoquer une autre fête bien plus tardive, celle organisée pour la fin de la captivité des princes français en Espagne, le $1^{\text {er }}$ juillet 1530. Feux de joie, musique et surtout « histoyres et morallitez » sont mis en place ${ }^{23}$. Une galère installée sur un grand char et tirée par quarante hommes porte entre autres trois enfants figurant les princes et trois filles représentant des vertus. Si rien n'est dit des décors eux-mêmes $^{24}$, de nombreux musiciens sont sollicités et un fatiste ${ }^{25}$, Bremond Dourat, est chargé de rédiger cent dictons, qui sont indiqués expressément comme devant être joués par deux fois ${ }^{26}$. Cette précision est exceptionnelle et n'apparaît jamais ailleurs, que ce soit pour les entrées ou les autres fêtes organisées à Lyon. Il semble donc que, dans la grande majorité des cas, les histoires n'aient été jouées qu'une fois, au seul bénéfice de l'hôte et de son proche entourage. Cette particularité distingue les entrées des mystères contemporains au cours desquels les scènes peuvent être jouées plusieurs fois ${ }^{27}$. À Paris en revanche certaines scènes semblent avoir été préparées pour le divertissement d'un grand nombre de personnes : en 1504, un personnage est payé pour expliquer

\footnotetext{
${ }^{23}$ A. M. Lyon, CC800, pièce $\mathrm{n}^{0} 1$ (7 juillet 1530).

${ }^{24}$ A. M. Lyon, CC800, pièce $\mathrm{n}^{\mathrm{o}} 1, \mathrm{f}^{\mathrm{o}} 3$ : il est payé 2 livres pour avoir « paint et doré visaiges d'or et d'argent les joueurs » et «les mores qui ont trainé le chariot et gallere $\gg$.

${ }^{25}$ Le terme de «fatiste » désigne des poètes; il apparaît à Lyon en 1515 pour désigner ceux en charge des entrées.

${ }^{26}$ A. M. Lyon, CC800, pièce $n^{\circ} 1, \mathrm{f}^{0} 3$. Ce poète n'est pas connu par ailleurs.

${ }^{27}$ C'est par exemple le cas pour la passion d'Arnoul Gréban ou la vie des saints Crépin et Crépinien, à Paris. Cf. Michel Rousse, La Scène et les tréteaux. Le théâtre de la farce au Moyen Âge, Orléans, Paradigme, coll. « Medievalia », nº 50, 2004.
} 
plusieurs fois un mystère de l'entrée d'Anne de Bretagne aux passants. Rien de tel à Lyon où on ne rencontre jamais cette fonction tenue par celui qu'on appelle souvent un « expositeur».

Outre les échafauds, plusieurs «joyeusetés » étaient organisées à des carrefours, cette fois par les habitants. Pour ce genre d'amusements, la participation du public est toute autre. Il s'agit par exemple des fontaines qui distribuent du vin ou dont l'eau brûle. On peut donc supposer que ces fontaines étaient en activité durant tout le temps de l'entrée, voire au-delà, et donc visibles et accessibles à tous, à l'inverse des grandes histoires.

Le seul document qui pourrait nous renseigner est le manuscrit de l'entrée de François I ${ }^{\mathrm{er}}$, le seul de ce type conservé pour la ville de Lyon. Dans le prologue, le narrateur indique que le roi arrive le 12 juillet 1515 vers quinze heures en ville. Suit la description minutieuse des histoires et du défilé dans les rues. Le texte se termine sur la simple indication que le roi entre dans la cathédrale où se déroule la cérémonie religieuse puis qu'il rejoint ensuite son logis où son souper l'attend. Malheureusement, cette indication n'est que peu significative, l'heure du souper pouvant se situer entre dix-sept et vingt heures. La durée de l'entrée reste donc impossible à déterminer avec certitude.

\section{Le prolongement de l'entrée}

Le caractère éphémère des entrées, donc limité dans le temps, en est un trait constitutif. Des décors, des costumes, des textes, il ne reste en général rien. Les histoires inventées pour la venue des rois ou des princes de passage ne sont jouées et vécues qu'une fois et jamais répétées. L'entrée présente pourtant de nombreux éléments répétitifs et peut trouver une sorte de prolongement dans le temps par le réemploi des 
éléments de décors ou encore grâce au souvenir qu'elle laisse dans la cité.

La trace d'œuvres pérennes dérivées des grands décors ou des histoires inventées pour ces occasions fait malheureusement défaut. Ce sont pourtant les mêmes artistes qui travaillent aux différentes catégories d'œuvres (vitraux, panneaux, décors éphémères). En revanche, on connaît plusieurs exemples de réutilisation des décors, soit pour des mystères, soit encore pour d'autres entrées.

Autre prolongement de la fête, le manuscrit qui en garde le souvenir. Les manuscrits d'entrée sont plutôt rares et apparaissent tous entre l'extrême fin du $X^{\mathrm{e}}$ siècle et le premier tiers $d u X V I^{e}$ siècle; ils sont rapidement supplantés par des livrets imprimés. Le déroulé de la fête, les paroles des personnages y sont reportés, accompagnés de riches enluminures qui, à l'inverse des livres contenant des pièces de théâtre, sont presque toujours en pleine page et non insérées dans les colonnes de texte. La question de la fidélité de ces manuscrits à ce que fut la fête se pose évidemment. Le discours est-il conforme à celui pensé par les fatistes et reçu par les spectateurs ${ }^{28}$ ? Pour le cas lyonnais le plus célèbre, le manuscrit de l'entrée de François I ${ }^{\mathrm{er}}$ (1515), la confrontation entre les miniatures, le texte du manuscrit et les archives révèle des différences notables. Le manuscrit est donc une recréation à partir de l'événement ; il le prolonge dans le temps sans en être la réplique exacte. En outre, il ne se trouvait pas dans les mains du roi quand celui-ci parcourait les rues de la ville. On en trouve la preuve dans le fait que la date et l'heure exactes soient indiquées au début du manuscrit, de la même encre et de la même main que le reste du texte, éléments qui pouvaient difficilement être connus à l'avance.

\footnotetext{
${ }^{28}$ Autant de questions posées par les auteurs s'intéressant aux livrets d'entrées imprimés.
} 
Les entrées présentent un schéma d'organisation récurrent, dont la durée s'étend souvent sur plusieurs semaines. Or l'invention des peintres et des notables doit être à l'inverse très rapide, surtout au début de la période étudiée ici. C'est la réalisation des décors et leur mise en place qui mobilise le temps des préparatifs et non leur conception, alors même que l'invention et la nouveauté sont recherchées et revendiquées par les artistes. Une fois le temps de l'entrée venu, le déroulé échappe quelque peu à notre regard : qui voit ces histoires, qui perçoit les textes récités ou chantés, qui contemple les effets spéciaux ? Ce n'est certainement qu'un petit nombre de personnes massées autour de l'hôte. Événement éphémère par nature, l'entrée est également éphémère dans son déroulement. Seules les archives et, à un moment tardif de l'histoire du phénomène, le manuscrit enluminé puis le livret, viennent prolonger cette fête, conservant un souvenir - bien que subjectif - de l'entrée et lui donnant ainsi une temporalité supplémentaire. 